\title{
Nuevos antifúngicos: Las equinocandinas
}

\author{
ALEXIS DIOMEDI P.
}

New antifungal agents: Echinocandins

The lipopeptide family known as echinocandins emerge as the new "antifungal penicillins", because their ability to destroy the fungal cell wall as they inhibit glucan synthesis, the main component of fungal structure. Echinocandins are fungicidal in vitro and in vivo against most Candida species and fungistatic against Aspergillus sp, without antifungal activity over mammal cells. Three drugs are representative of this class; caspofungin, micafungin and anidulafungin, the two first have been licensed for human use. Their optimal security profile, with low incidence and severity of adverse effects, kind posology and few interactions with other drugs, represent noticeable advantages for modern antifungal therapy. They have similar clinical efficacy as amphotericin B, without its toxicity, which besides the absence of antagonism with other antifungal drugs, allows to suggest that combined antifungal therapy could represent a new standard for the management of the feared invasive aspergillosis.

Key words: Echinocandins; Caspofungin; Micafungin; Anidulafungin.

Palabras claves: Equinocandinas; Caspofungina; Micafungina; Anidulafungina.

\section{Introducción}

En las últimas décadas asistimos a la emergencia de los hongos como patógenos oportunistas humanos. Sobre 7\% de los pacientes en hospitales universitarios europeos fallecen de aspergilosis y diversas especies de Candida se han constituido en causa común de infección nosocomial ${ }^{1,2}$. Ciertos grupos de pacientes inmunocomprometidos presentan alto riesgo de micosis severas; por ejemplo, $15 \%$ de los receptores de trasplante alogeneico de células madres ${ }^{3}$, y casi $20 \%$ de los sometidos a trasplantes pulmonares, están colonizados o infectados ${ }^{4}$; en los pacientes con SIDA de países desarrollados la neumonía por Pneumocystis jiroveci (ex P. carinii) y la candidiasis esofágica alcanzan incidencias de 60 y $20 \%$ respectivamente 5 . En Chile, Lucero y cols reportan $9,2 \%$ de infecciones fúngicas profundas en niños con episodios de neutropenia febril ${ }^{6}$; por otro lado, Wolff y cols ${ }^{7}$ reportan que en el seguimiento de una cohorte de 166 pacientes adultos con infección por VIH y sin terapia antiretroviral, 17,2\% por año presentó candidiasis esofágica, $9,1 \%$ por año neumonía por $P$. jiroveci y $4,1 \%$ por año criptococosis meníngea.
Diversos factores dan cuenta de este aumento en las infecciones fúngicas, a saber, el mejor manejo de otras complicaciones de la inmunodepresión, nuevos y cada vez más agresivos regímenes inmunosupresores, mayor sobrevida en pacientes de unidades de cuidados críticos, alta incidencia de procedimientos invasores y cateterizaciones, mayor sospecha clínica, mejores métodos diagnósticos y el uso exagerado de antimicrobianos.

A la fecha, el manejo de las infecciones micóticas sistémicas se sostiene en tres grupos de antifúngicos; polienos (anfotericina B), azoles (itraconazol, fluconazol, voriconazol, entre otros) y la fluocitosina (no disponible en Chile) (Tabla 1). A pesar del importante aporte de estos fármacos al manejo de las infecciones fúngicas, los índices de fracaso elevados ${ }^{4}$, la presencia de efectos adversos significativos y la emergencia de especies fúngicas intrínsecamente o secundariamente resistentes, son problemas crecientes y no cabalmente resueltos ${ }^{8,9}$. De allí que la aparición de esta nueva clase terapéutica, las equinocandinas, es bienvenida. El objetivo de esta revisión es examinar sistemáticamente los datos científicos disponibles que avalan el uso clínico ac-

Hospital Del Salvador e Instituto Nacional del Tórax.

Recibido: 19 enero 2004

Aceptado: 7 abril 2004 
Tabla 1. Mecanismos de acción de antifúngicos e implicaciones para su eficacia (Modificado de ref 19)

\begin{tabular}{|c|c|c|c|}
\hline $\begin{array}{l}\text { Agente } \\
\text { antifúngico }\end{array}$ & $\begin{array}{l}\text { Sitio de acción en } \\
\text { la célula fúngica }\end{array}$ & Actividad & Implicaciones clínicas \\
\hline Polienos & Membrana & $\begin{array}{l}\text { Se une a ergosterol causando la } \\
\text { muerte celular }\end{array}$ & $\begin{array}{l}\text { Potente actividad antifúngica de } \\
\text { amplio espectro }\end{array}$ \\
\hline Azoles & Membrana & $\begin{array}{l}\text { Inhibe la enzima CYP } 450 \\
\text { responsable de la síntesis de } \\
\text { ergosterol, daña la membrana } \\
\text { citoplasmática }\end{array}$ & $\begin{array}{l}\text { Actividad fungistática de } \\
\text { potencia y espectro variable }\end{array}$ \\
\hline Fluocitosina & Núcleo & $\begin{array}{l}\text { Interfiere con la síntesis del ADN } \\
\text { al inhibir timidilato sintetasa }\end{array}$ & $\begin{array}{l}\text { Espectro restringido. Actividad } \\
\text { antifúngica sumatoria en combi- } \\
\text { nación con anfotericina B }\end{array}$ \\
\hline Equinocandinas & Pared celular & $\begin{array}{l}\text { Inhibe la síntesis de glucano, } \\
\text { destruye la estructura de la pared } \\
\text { celular causando lisis fúngica }\end{array}$ & $\begin{array}{l}\text { Potente actividad antifúngica de } \\
\text { amplio espectro. Potencial efecto } \\
\text { aditivo en terapias combinadas }\end{array}$ \\
\hline
\end{tabular}

tual y futuro de las equinocandinas licenciadas o próximas a ser licenciadas para su uso en seres humanos.

\section{Metodología de búsqueda y criterios de selección}

Se revisaron todas las referencias citadas en MEDLINE a diciembre de 2003, considerando términos claves como Echinocandins, Caspofungin, Micafungin y Anidalufungin. Se incluyó además la información de los resúmenes de conferencias como 43rd Interscience Conference on Antimicrobial Agents and Chemotherapy (ICACC), el $41^{\text {th }}$ Meeting of Infectious Diseases Society of America (IDSA) y el $9^{\text {th }}$ Congress of European Confederation of Medical Mycology (ECMM), todos realizados durante 2003. Se obtuvo reseña adicional de documentos de acceso libre en Internet y de la base de datos farmacéutica comercial MICROMEDEX.

\section{El génesis de las equinocandinas}

Las equinocandinas son lipopéptidos sintéticamente modificados, derivados originalmente de la fermentación de caldos de diferentes hongos. El componente mayor de la anidulafungina (LY303366; Figura 1) se identificó en $1974^{10}$. En 1989, se reportó el precursor de caspofungina $(\text { MK991) })^{11}$ y en 1990 el de micafungina (FK463) ${ }^{12}$. Se han descrito varios otros análogos y derivados de equinocandinas; la enfumafungina, las arbocandinas, las papulacandinas, la pneumocandina $\mathrm{B}$, la arundifungina y la mulucandina ${ }^{13-17}$. Otras como la cilofungina han sido abandonadas en su desarrollo clínico dada su difícil formulación y gran toxicidad.

\section{Mecanismos de acción}

El blanco de las equinocandinas es el complejo de síntesis de la pared celular fúngica $\beta-1,3-\mathrm{D}$ glucano sintetasa ${ }^{18}$. En base a tal mecanismo se ha planteado una homología con penicilina y sus derivados que actúan sobre transpeptidasas y sintetasas en la pared bacteriana (PBP), por lo que algunos autores insinúen que las equinocandinas serán las "penicilinas antifúngicas". Dado que las células de mamíferos carecen de pared celular, este blanco de acción es óptimo para los antifúngicos. Un diagrama de la pared celular fúngica y sus componentes se muestra en la Figura 2. La depleción de glucano de la pared fúngica consecuente lleva a un colapso osmótico, balonamiento y finalmente lisis de la célula fúngica ${ }^{19}$.

\section{Aspectos farmacológicos}

Las equinocandinas son macro-lipopéptidos. Las moléculas actualmente en uso o en desarrollo son hexapéptidos cíclicos anfífilicos con una cadena lateral aminoacil-lipídica y un peso molecular de casi 1.200 daltons ${ }^{14,20}$. Sus estructuras se ven en la Figura 1. La caspofungina tiene un ácido graso como cadena lateral, micafungina una complejo aromático 3,5-difenilisoxasol substituido, y anidulafungina una cadena alcoxitrifenilo. Se supone que esta cadena lateral se introduce en la bicapa lipídica de la membrana celular fúngica. Caspofungina es fácilmente soluble en agua y metanol y levemente soluble en etanol. Micafungina es fácilmente soluble en agua, pero anidulafungina no.

Las preparaciones de equinocandinas son de uso endovenoso exclusivo. Caspofungina se en- 
cuentra licenciada en E.U.A., Europa y es la única equinocandina actualmente disponible en nuestro país (Tabla 2). Micafungina esta licenciada en Japón y espera su licenciamiento en E.U.A. para fines de 2004. Estos antifúngicos poseen características farmacocinéticas comunes (Tabla 2), como por ejemplo su escasa biodisponibilidad oral, menor de $0,2 \%$ para caspofungina $^{21}$. En un estudio en voluntarios con SIDA dosis orales sobre $500 \mathrm{mg}$ diarios de anidulafungina, permitieron alcanzar concentraciones plasmáticas pico de $753 \mathrm{ng} / \mathrm{ml}$, lo que sin embargo, fue insuficiente para obtener resultados adecuados en el tratamiento de la candidiasis oro-faríngea ${ }^{20}$.

Todas las equinocandinas tienen una cinética lineal luego de la administración endovenosa. Una de sus características distintivas es su unión a proteínas (Tabla 3) que pudiera limitar la cantidad de fármaco activo disponible. Aún así, sólo una pequeña cantidad de micafungina está unida en forma covalente a albúmina. Diversos antifúngicos están altamente unidos a proteínas, incluyendo anfotericina B e itraconazol, a diferencia

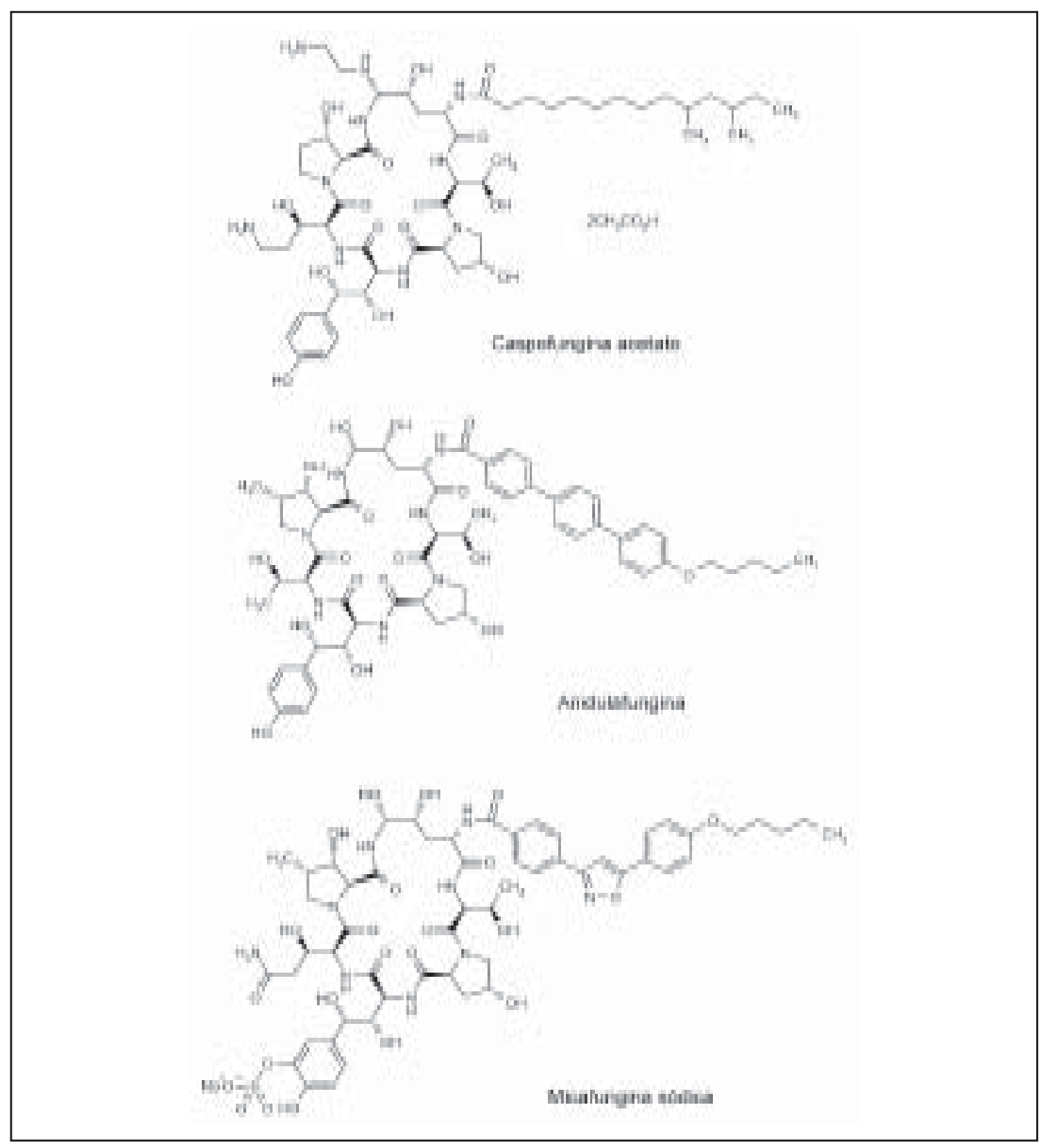

Figura 1. Estructura química de caspofungina, micafungina, y anidulafungina. (Modificado de ref. 73). 


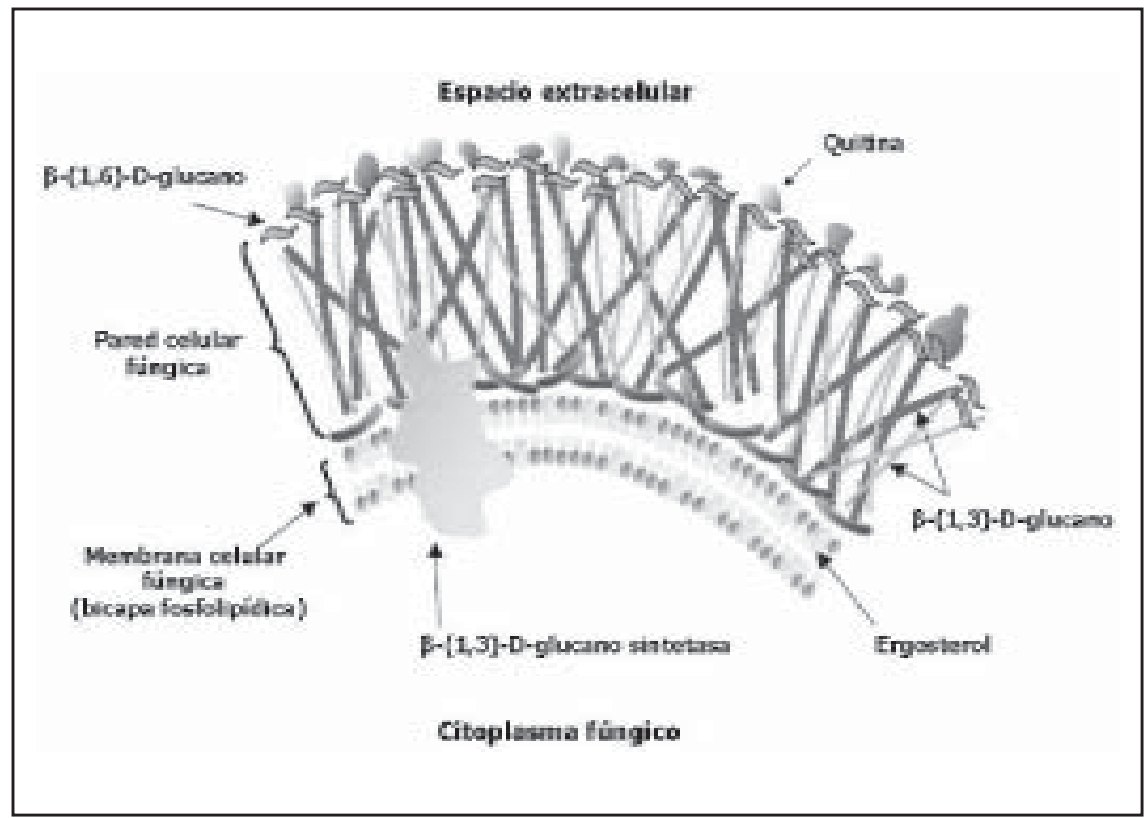

Figura 2. Diagrama de la membrana celular fúngica. (Modificado de ref. 74).

Tabla 2. Equinocandinas y su estado de desarrollo

\begin{tabular}{lcc}
\hline Fármaco & Fabricante & Estado de desarrollo actual \\
\hline Caspofungina (MK991, Cancidas $\left.{ }^{\circledR}\right)$ & Merck & Aprobada en E.U.A. y Europa. \\
Micafungina (FK463, Funguard®, Mycamine $\left.{ }^{\circledR}\right)$ & Fujisawa & Aprobada en Japón. \\
Anidulafungina (LY303366, VER002, V-Candin $\left.{ }^{\circledR}\right)$ & Vicuron & Fase 3 \\
Mulucandina (HMR 3270) & Indevus & Fase 1 \\
\hline
\end{tabular}

Tabla 3. Variables farmacocinéticas relevantes de las equinocandinas en el uso clínico

\begin{tabular}{|c|c|c|c|}
\hline & Caspofungina & Micafungina & Anidulafungina \\
\hline \multicolumn{4}{|l|}{ Variable } \\
\hline Cmax a $70-75$ mg/día $(\mu \mathrm{g} / \mathrm{ml})^{*}$ & $12,1(11,1-13,2)$ & 10,9 (DS 1,5) & 3,44 \\
\hline $\mathrm{AUC}_{0-24}$ (estado estable) $\left(\mu \mathrm{g} \mathrm{h}^{-1} \mathrm{ml}^{-1}\right)$ & $100,5(87,9-114,8)$ & $111,3(\mathrm{DS} 14,1)$ & 44,4 \\
\hline Beta $t_{1 / 2}(h)$ & $10,6(\mathrm{SD} 1,1)$ & $11-17$ & 18,1 \\
\hline \multirow[t]{2}{*}{ Rango terapéutico plasmático $(\mu \mathrm{g} / \mathrm{ml})$} & $1-2$ & Candidiasis $0,02-2$ & 0,5 \\
\hline & & Aspergillosis 0,02 & \\
\hline Aclaramiento (mL/min) & $10-12,5$ & $\sim 10,5$ & $12,5-19,2$ \\
\hline Volumen de distribución (1/kg) & $\dagger$ & 0,26 & 0,57 \\
\hline $\mathrm{t}_{1 / 2}$ en falla hepática $(\mathrm{h})$ & NA (prolongada) & $14,4$ (DS 0,8$)$ & $34-42$ \\
\hline$t_{1 / 2}$ en falla renal severa $(h)$ & $\mathrm{NA} \neq$ & $14,2(\mathrm{DS} 1,5)$ & $33-42$ \\
\hline Unión a proteínas & $96 \%$ & $99,8 \%$ & $84 \%$ \\
\hline Concentración urinaria (\% del plasma) & $1,4 \%$ & $0,7 \%$ & $<0,1 \%$ \\
\hline Concentración en LCR (\% del plasma) & ¿bajo? & ¿bajo? & $<0,1 \%$ \\
\hline
\end{tabular}

Los datos se expresan en promedio (DS), mediana (IQR), rango, o porcentaje. Estos datos son derivados de diferentes fuentes y ensayos, por lo tanto no siempre son directamente comparables.

$\mathrm{NA}=$ no disponible. $\mathrm{t}_{1 / 2}=$ vida media.

* Anidulafungina dosis de carga de $150 \mathrm{mg}$, seguida de $75 \mathrm{mg}$ diarios, caspofungina $70 \mathrm{mg} /$ diarios.

† Datos no disponibles porque la captación de los tejidos es compleja y está en investigación.

\$ Probablemente similar a los voluntarios sanos. 
de moléculas hidrosolubles como fluconazol y fluocitosina. Aunque las concentraciones en LCR de anfotericina e itraconazol son bajas, ambos fármacos son eficaces ante meningitis fúngicas ${ }^{22}$. De lo anterior se desprende que la importancia de la unión de estas moléculas con proteínas no es clara. La degradación de las equinocandinas se lleva a cabo principalmente en el hígado por mecanismos de hidrólisis y amino-acetilación ${ }^{21}$. La captación hepática y degradación es lenta, sobre todo para caspofungina y micafungina, llevando a una larga vida media final. La excreción se produce lentamente por la bilis. Estos fármacos no son dializables, por lo que no requieren ajuste en falla renal sometida a hemodiálisis. Para caspofungina se recomienda reducir la dosis diaria a la mitad, luego de una dosis estándar de carga, en pacientes con falla hepática severa, aunque no existe respaldo clínico para tal recomendación ${ }^{23}$.

No existe experiencia acumulada acerca del uso de caspofungina en niños; sin embargo, algunos datos sugieren que se pudiera necesitar una dosis mayor comparada con la de adul$\operatorname{tos}^{21,24}$. Micafungina, en cambio, sí ha sido ampliamente evaluada en niños (salvo prematuros) siendo su farmacocinética similar a la de los adultos ${ }^{25}$.

\section{Interacciones con otros fármacos}

Dada su escasa metabolización por el sistema de citocromos $\mathrm{P} 450$, las equinocandinas presentan muy pocas interacciones medicamentosas comparadas con los azoles. El aclaramiento de caspofungina se ve aumentado por fármacos como carbamazepina, dexametasona, efavirenz, nelfinavir, nevirapina, fenitoina y rifampicina ${ }^{26}$. Un efecto inverso tiene ciclosporina que al retardar su aclaramiento, aumenta las concentraciones plasmáticas de caspofungina ${ }^{21}$. Por otro lado, el uso conjunto de caspofungina con tacrolimus redunda en un aumento de la excreción de este último fármaco ${ }^{27}$. No se han reportado interacciones relevantes de caspofungina con anfotericina B, itraconazol ni con micofenolato ${ }^{21,28}$.

No se han descrito interacciones importantes de micafungina con warfarina, ácido acetilsalicílico, diazepam ni metrotrexato. Tampoco se han evidenciado interacciones con ciclosporina ni con tacrolimus ${ }^{29}$. El uso combinado de anidulafungina con ciclosporina determina un aumento en las concentraciones plasmáticas de esta equinocandina ${ }^{29,30}$.

\section{Espectro antifúngico}

El especto antifúngico de las equinocandinas esta circunscrito a Candida spp y Aspergillus spp (Tabla 4). Los tres antifúngicos son fungicidas in vitro e in vivo contra la mayoría de las cepas de Candida y fungistáticas contra Aspergillus ${ }^{31}$. No se ha descrito resistencia cruzada con polienos ni azoles, siendo poco probable que aparezca dado su mecanismo de acción particular. Denning ${ }^{31}$ realizó una revisión acabada sobre la evaluación in vitro del espectro del antifúngico de las equinocandinas.

Tabla 4. Espectro de actividad de las equinocandinas (Modificado de ref. 31)

\begin{tabular}{llll}
\hline Actividad alta & Actividad adecuada & Actividad baja & Inactivas \\
\hline Candida albicans & Candida parapsilosis & Coccidioides immitis & Zygomycetes \\
Candida glabrata & Candida guilliermondi & Blastomyces dermatitidis & Cryptococcus neoformans \\
Candida tropicalis & Aspergillus fumigatus & Scedosporium spp & Fusarium spp \\
Candida krusei & Aspergillus flavus & Paecilomyces variotii & Trichosporon spp \\
Candida kefyr & Aspergillus terreus & Histoplasma capsulatum & \\
Pneumocystis jiroveci* & Candida lusitaniae & & \\
\hline Actividad alta implica CIM & & \\
\hline
\end{tabular}

Actividad alta implica CIM muy bajas con acción fungicida y buena acción in vivo, Actividad adecuada implica CIM bajas, pero sin acción fungicida en la mayoría de los casos, Actividad baja implica acción detectable, que pudiera tener potencial terapéutico en el hombre (en algunos casos combinadas con otros antifúngicos). Inactiva implica sin acción intrínseca.

* Sólo activas frente a formas quísticas, probablemente sólo útil en profilaxis. 


\section{Estudios clínicos}

El desarrollo clínico de las equinocandinas se ha orientado hacia indicaciones apropiadas para un fármaco endovenoso, incluyendo candidiasis esofágica, candidiasis invasora y candidemia, aspergilosis invasora, y profilaxis de infecciones fúngicas invasoras. Los ensayos clínicos iniciales comparaban anfotericina B con tres diferentes dosis de caspofungina en el tratamiento de candidiasis esofágica en pacientes con SIDA (Tabla 5) ${ }^{32,33}$. Caspofungina a dosis diaria de $35 \mathrm{mg}$ es menos activa que 50 y $70 \mathrm{mg}$ diarios, además estas últimas dosis son mejor que anfotericina $\mathrm{B}$ en dosis de $0,5 \mathrm{mg} / \mathrm{kg}$, aunque el intervalo de confianza es ancho ${ }^{32,33}$. Caspofungina $35 \mathrm{mg}$ diarios fue equivalente a fluconazol $200 \mathrm{mg}$ diarios. Los resultados de un estudio fase 2 de comparación de dosis con micafungina (25-100 mg diarios) en 120 pacientes con candidiasis esofágica, mostraron que la mejor respuesta clínica se consiguió con dosis diarias mayores de $25 \mathrm{mg}$ diarios, y mejor respuesta endoscópica con dosis mayores de $75 \mathrm{mg}$ diarios $^{34}$. Las tasas de respuesta global fueron similares a las vistas con fluconazol ${ }^{35}$. También se han visto buenos resultados con dos dosis de anidulafungina en candidiasis esofágica demostrada endoscópicamente $^{36}$. Un reciente estudio randomizado de anidulafungina y fluconazol en 601 pacientes mostró eficacia clínica similar entre ambos fármacos en candidiasis esofágica ${ }^{37-39}$. En los ensayos donde fluconazol ha fracasado por resistencia, se ha visto buenas tasas de respuesta con caspofungina ${ }^{40} \mathrm{y}$ anidulafungina ${ }^{41}$. Estos resultados muestran claramente la eficacia de las equinocandinas en el tratamiento de infecciones fúngicas severas de mucosas.

En un estudio aleatorio de terapia primaria de candidiasis invasora y candidemia, la terapia con caspofungina fue equivalente a anfotericina $\mathrm{B}^{42}$. La dosis de caspofungina utilizada fue la habitual carga de $70 \mathrm{mg}$ seguida de $50 \mathrm{mg}$ diarios, comparándose este régimen con anfotericina $\mathrm{B}(0,6-1$ $\mathrm{mg} / \mathrm{kg}$ ). Las tasas de respuesta en ambas ramas del estudio fueron 76 y $78 \%{ }^{42}$ consideradas favorables al compararlas con datos previos de fluconazol y anfotericina $\mathrm{B}^{43,44}$. Caspofungina fue menos tóxica que anfotericina $B$, siendo efectiva para todas las especies de candida. Un estudio abierto extenso de micafungina para la misma indicación (pero mayoritariamente en pacientes intolerantes o fracasando a otros tratamientos) ha concluido recientemente ${ }^{45}$. A la fecha se enrolaron 173 pacientes, 119 con candidemia y 54 con candidiasis invasora. Algunos recibieron terapia primaria con micafungina, otros terapia de rescate, y otros combinación con otros antifúngicos, usualmente anfotericina B lipídica. Los objetivos finales a evaluar fueron determinados por un experto externo. La tasa global de respuesta fue de $83 \%$ en pacientes con candidemia y $63 \%$ en candidiasis invasora (Tabla 6$)^{45}$. Un estudio fase 2 comparativo de dosis de anidulafungina (Tabla 7) completó el enrolamiento de 120 pacientes con tasas de respuesta entre 81 y $89 \%$, presentándose escasos eventos adversos ${ }^{46,47}$. Otro reciente estudio doble ciego de anidulafungina vs fluconazol en candidiasis esofágica, que reclutó 504 pacientes evaluables al concluir la terapia, demostró éxito en 97,2 y 98,8\%, respectivamente, en la evaluación endoscópica ${ }^{37-39}$. Los eventos adversos tuvieron similar frecuencia y perfil en los dos grupos de comparación.

En estos estudios, no se han mostrado diferencias significativas entre diferentes especies de Candida causantes de patología, lo que sugiere que la mayor CIM de las equinocandinas a $C$. parasilopsis $(0,5-2 \mu \mathrm{g} / \mathrm{l}$ vs 0,04-0,015 $\mu \mathrm{g} / \mathrm{l}$ de $C$. albicans) sería clínicamente insignificante. Tales datos necesitan cotejarse en pacientes con candidiasis más complejas, por ejemplo endocarditis o infecciones persistentes en neutropénicos. Actualmente no se dispone de detalles sobre su respuesta en los llamados "santuarios farmacológicos" como son el ojo, las vegetaciones, el mediastino y las meninges. Al respecto una reciente comunicación de Kartsonis y cols ${ }^{48}$, basada en una revisión de la base de datos de Merck en los ensayos previos al registro de caspofungina, sugiere que el fármaco sería eficaz en la terapia de ITU por Candida sp, con 11/12 respuestas favorables. Por otro lado, reportes anecdóticos muestran éxitos de la terapia con caspofungina en candidiasis hepatoesplénica resistente a anfotericina B liposomal ${ }^{49}$, infección protésica de cadera por C. glabrata $^{50}$ y endocarditis por C. albicans ${ }^{51}$. Algunos autores plantean que dado el amplio especto de acción sobre Candida, su rápida acción fungicida y los buenos resultados las equinocandinas llegarían a ser el tratamiento de elección para candidiasis invasora y candidemia.

Respecto de la aspergilosis, Merck desarrolló un estudio con caspofungina ${ }^{52}$ en el cual aquellos pacientes con falla a terapia a anfotericina $\mathrm{B}$, anfotericina $\mathrm{B}$ lipídica o itraconazol, más algunos pacientes con intolerancia a anfotericina $B$, se sometieron a caspofungina como fármaco único (Tabla 5). El estudio mostró una buena respuesta, en $45 \%$ de los pacientes, comprobando que caspofungina es activa en aspergilosis invasora ${ }^{52}$. 
Tabla 5. Principales estudios clínicos relativos a eficacia de caspofungina

\begin{tabular}{lcccccc}
\hline Diseño del & $\begin{array}{c}\text { Enfermedad } \\
\text { estudio }(\text { ref })\end{array}$ & $\begin{array}{c}\text { Número de } \\
\text { pacientes }\end{array}$ & $\begin{array}{c}\text { Dosis } \\
\text { diaria } \\
(\mathrm{mg})\end{array}$ & $\begin{array}{c}\text { Comparador } \\
\text { Dosis diaria }\end{array}$ & Respuesta & Comentarios \\
\hline
\end{tabular}

\begin{tabular}{llccc}
\hline $\begin{array}{l}\text { Terapia primaria, } \\
\text { comparación de } \\
\text { dosis, doble ciego }\end{array}$ & $\begin{array}{c}\text { Candidiasis } \\
\text { esofágica }\end{array}$ & 128 & 50 y $70 \mathrm{AmB} 0,5 \mathrm{mg} / \mathrm{kg}$ & $50 \mathrm{mg}(85 \%)$, \\
& & $70 \mathrm{mg}(96 \%), y$
\end{tabular}

Terapia primaria, Candidiasis comparación de orofaríngea y dosis, doble ciego esofágica (33)

Terapia primaria, Candidiasis doble ciego (65) esofágica

\section{Terapia de} rescate $(52)$

Doble-ciego (42)

Aspergilosis invasora

\section{3}
35, 50, AmB 0,5 mg/kg y 70

177

90

$$
\begin{aligned}
& \text { Candidiasis } \\
& \text { invasora }
\end{aligned}
$$

Terapia de rescate, Aspergilosis retrospectivo (66) invasora, candidiasis esofágica e invasora

Terapia de rescate, Aspergilosis prospectivo (67) invasora

$\begin{array}{lc}\text { Terapia profilác- } & \text { Profilaxis en } \\ \text { tica, doble ciego } & \text { NFP }\end{array}$
(68)

Terapia profilácti- Profilaxis en ca, estudio abierto, LMA y SMD randomizado (69) sometidos a QTI $200 \mathrm{mg}$

No
AmBL $3 \mathrm{mg} / \mathrm{kg}$

23

48

70
(carga)

luego 50

1.095

70

(carga) luego 50

192

50

$$
\begin{array}{r}
\text { ITRA } 200 \\
\text { cada } 12 \mathrm{~h} \\
\text { días, luego } \\
\text { mg ITRA } \\
\text { o caspofung } \\
\text { No } \\
\end{array}
$$

12

$$
\begin{gathered}
70 \\
\text { (carga) } \\
\text { luego } \\
50
\end{gathered}
$$

$35 \mathrm{mg}(74 \%)$ $50 \mathrm{mg}(91 \%)$, $70 \mathrm{mg}(78 \%) \mathrm{y}$ AmB (63\%)

85 vs $86 \%$ $37 / 83(45 \%)$

Dosis baja de AmB, las tasas de respuesta al final del tratamiento combinan criterios clínicos y endoscópicos

Dosis baja de AmB, verificación endoscópica de respuesta

Mayor tasa de recaída con caspofungina (28 vs $17 \%, \mathrm{p}=0,19)$

Alta proporción de fallas de terapias previas

83/109 (76\%) Más efectos tóxicos con vs $90 / 115(78 \%)$ anfotericina $\mathrm{B}$, respuesta similar en Candida albicans y no albicans

Global 63,6\% Uso compasivo, no hubo (14/22), 9/15 abandono por efectos aspergilosis, adversos $5 / 6$ candidiasis
9/45 (20\%) completo, $11 / 45(24 \%)$ parcial
Uso compasivo, 1 aban- dono por anafilaxis

Éxito global ITT Similar resultado en re33,9 vs $33,7 \%$ solución de la fiebre
11/12 con uro- cultivo control negativo al final del tratamiento
Revisión base de datos Merck, incluye uso com- pasivo ( $3 \mathrm{p}$ ) y estudio de candidiasis invasora (9 p)

$\mathrm{AmB}=$ anfotericina $\mathrm{B} . \mathrm{AmBL}=$ anfotericina $\mathrm{B}$ liposomal, $\mathrm{NFP}=$ neutropenia febril persistente, $\mathrm{LMA}=$ leucemia mieloide aguda, $\mathrm{SMD}=$ síndrome mielodisplásico, QTI= quimioterapia de inducción, ITT= intención de tratar, ITRA= itraconazol, ITU= infección tracto urinario. 
Se vio mejor resultado en los pacientes intolerantes a anfotericina $\mathrm{B}$ que en los que fallaron al tratamiento, como podría esperarse, mostrando ese pequeño grupo una tasa de respuesta de $75 \%$. Dado que tanto anfotericina B como itraconazol tienen vidas medias prolongadas, la respuesta inicial pudiera ser consecuencia de un breve periodo de "terapia combinada", que se continuara con los buenos efectos de caspo- fungina. Otro punto crucial que cuestiona estos estudios sobre aspergilosis invasora se refiere a la rapidez con que muchos de estos pacientes fallecen, situación que impide que sean enrolados en los ensayos, puesto que no sobreviven lo suficiente para ser incluidos, o son excluidos por su gravedad. De esta manera se estaría sobreestimando la respuesta favorable en relación a la población general con tal patología.

Tabla 6. Principales estudios clínicos relativos a eficacia de micafungina

\begin{tabular}{lcccccc}
\hline $\begin{array}{l}\text { Diseño del } \\
\text { estudio (ref) }\end{array}$ & $\begin{array}{c}\text { Enfermedad } \\
\text { fúngica }\end{array}$ & $\begin{array}{c}\text { Número de } \\
\text { pacientes }\end{array}$ & $\begin{array}{c}\text { Dosis } \\
\text { diaria } \\
(\mathbf{m g})\end{array}$ & $\begin{array}{c}\text { Comparador } \\
\text { Dosis diaria }\end{array}$ & Respuesta & Comentarios \\
\hline $\begin{array}{l}\text { Terapia profilác- } \\
\text { tica, doble ciego }\end{array}$ & $\begin{array}{c}\text { Profilaxis en } \\
\text { TCMH }\end{array}$ & 882 & 50 & $\begin{array}{c}\text { Fluconazol } \\
400 \mathrm{mg}\end{array}$ & $\begin{array}{c}80 \mathrm{vs} 73,5 \% \\
(\mathrm{p}=0,025)\end{array}$ & $\begin{array}{l}\text { Extenso estudio con be- } \\
\text { neficio en todas las po- } \\
\text { blaciones y subgrupos } \\
\text { (60) }\end{array}$ \\
& & & & & incluidos
\end{tabular}

Terapia primaria, Candidiasis $119 \quad$ 12,5, No $\quad$ Tasa de respuesta Peor respuesta en padosis comparativo esofágica $\quad 25$ y $50 \quad$ endoscópica; $81 \%$ cientes con enfermedad (34) dosis $12,5 \mathrm{mg}$ /día, severa $97,2 \%$ con dosis $>50 \mathrm{mg} /$ día y

$>10$ días de terapia

\begin{tabular}{|c|c|c|c|c|c|c|}
\hline $\begin{array}{l}\text { Terapia primaria } \\
\text { y de rescate, } \\
\text { estudio abierto } \\
(70)\end{array}$ & $\begin{array}{c}\text { Candidiasis } \\
\text { esofágica }\end{array}$ & 97 & 50 y 100 & No & $\begin{array}{l}92 \% \text { respuesta } \\
\text { completa o parcial. } \\
78 \% \text { en infección } \\
\text { refractaria }\end{array}$ & $\begin{array}{l}4 \text { pacientes pediátricos. } \\
\text { Buena respuesta tanto } \\
\text { para } C \text {. albicans como } \\
\text { no albicans }\end{array}$ \\
\hline $\begin{array}{l}\text { Terapia primaria, } \\
\text { de rescate, y } \\
\text { combinación }(45)\end{array}$ & $\begin{array}{l}\text { Candidiasis } \\
\text { invasora o } \\
\text { candidemia }\end{array}$ & 142 & $50-100$ & No & $\begin{array}{l}83 \% \text { candidemia, } \\
63 \% \text { candidiasis } \\
\text { invasora }\end{array}$ & $\begin{array}{l}\text { Tasa de respuesta simi- } \\
\text { lar para todas las espe- } \\
\text { cies, menor para terapia } \\
\text { de rescate }\end{array}$ \\
\hline $\begin{array}{l}\text { Terapia primaria, } \\
\text { estudio abierto } \\
(57)\end{array}$ & $\begin{array}{l}\text { Candidiasis, } \\
\text { aspergilosis }\end{array}$ & 70 & $12,5-150$ & No & $\begin{array}{c}\text { Respuesta clínica; } \\
\text { candidemia (100\%), } \\
\text { candidiasis esofá- } \\
\text { gica }(71 \%), \text { API } \\
(60 \%), \text { APCN } \\
(67 \%) \text {, aspergi- } \\
\text { loma }(55 \%)\end{array}$ & $\begin{array}{l}\text { El amplio rango de dosis } \\
\text { y la heterogeneidad de } \\
\text { las infecciones dificultan } \\
\text { la evaluación }\end{array}$ \\
\hline $\begin{array}{l}\text { Terapia de rescate, } \\
\text { combinación } \\
(55,56)\end{array}$ & $\begin{array}{c}\text { Aspergilosis } \\
\text { invasora }\end{array}$ & 290 & $50-100$ & No & $37 \%$ & $\begin{array}{l}\text { La mayoría tratado en } \\
\text { combinación con AmB, } \\
\text { población de mal pro- } \\
\text { nóstico }\end{array}$ \\
\hline $\begin{array}{l}\text { Terapia primaria, } \\
\text { dosis comparativo, } \\
\text { doble ciego }(71,72)\end{array}$ & $\begin{array}{c}\text { Candidiasis } \\
\text { esofágica }\end{array}$ & 245 & $\begin{array}{c}50,100 \\
\text { y } 150\end{array}$ & $\begin{array}{l}\text { Fluconazol } \\
200 \mathrm{mg}\end{array}$ & $\begin{array}{c}50 \mathrm{mg}(71,2 \%), \\
100 \mathrm{mg}(91,7 \%), \\
150 \mathrm{mg}(98 \%), \\
\text { Flu }(95,8 \%) \mathrm{en} \\
\text { pacientes con } \\
\text { endoscopia inicial } \\
\text { y al final tratamient }\end{array}$ & $\begin{array}{l}\text { Verificación endoscópica } \\
\text { de respuesta, similar } \\
\text { perfil efectos adversos } \\
\text { o }\end{array}$ \\
\hline
\end{tabular}

$\mathrm{AmB}=$ anfotericina $\mathrm{B}, \mathrm{Flu}=$ fluconazol. $\mathrm{TCMH}=$ trasplante de células madres hematopoyéticas. API= aspergilosis pulmonar invasora; $\mathrm{APCN}=$ aspergilosis pulmonar crónica necrosante. 
Tabla 7 Principales estudios clínicos relativos a eficacia de anidulafungina

\begin{tabular}{|c|c|c|c|c|c|c|}
\hline $\begin{array}{l}\text { Diseño del } \\
\text { estudio (ref) }\end{array}$ & $\begin{array}{l}\text { Enfermedad } \\
\text { fúngica }\end{array}$ & $\begin{array}{l}\text { Número de } \\
\text { pacientes }\end{array}$ & $\begin{array}{c}\text { Dosis } \\
\text { diaria } \\
(\mathrm{mg})\end{array}$ & Comparador & Respuesta & Comentarios \\
\hline $\begin{array}{l}\text { Terapia primaria, } \\
\text { dosis comparativo } \\
(36)\end{array}$ & $\begin{array}{l}\text { Candidiasis } \\
\text { esofágica }\end{array}$ & 36 & $\begin{array}{c}50 \\
\text { (carga) } \\
\text { luego } 25 \\
\text { y } 70 \\
\text { (carga) } \\
\text { luego } 50\end{array}$ & No & $\begin{array}{c}\text { Tasa de respuesta } \\
\text { endoscópica } 81 \% \\
(50 / 25) \text { y } 85 \% \\
(70 / 35)\end{array}$ & $\begin{array}{l}\text { Respuesta clínica leve- } \\
\text { mente mayor en el gru- } \\
\text { po de dosis más alta }\end{array}$ \\
\hline $\begin{array}{l}\text { Terapia primaria, } \\
\text { doble ciego } \\
(37,38,39)\end{array}$ & $\begin{array}{l}\text { Candidiasis } \\
\text { esofágica }\end{array}$ & 601 & $\begin{array}{c}100 \\
\text { (carga) } \\
\text { y luego } \\
50\end{array}$ & $\begin{array}{c}\text { Fluconazol } \\
200 \mathrm{mg} \text { (carga) } \\
\text { y luego } \\
100 \mathrm{mg} / \text { día }\end{array}$ & $\begin{array}{l}\text { Éxito endoscópico } \\
97,2 \text { vs } 98,8 \%\end{array}$ & $\begin{array}{l}504 \text { pacientes evaluables } \\
\text { al final de tratamiento. } \\
\text { Perfil de seguridad simi- } \\
\text { lar }\end{array}$ \\
\hline $\begin{array}{l}\text { Terapia primaria, } \\
\text { abierto, dosis } \\
\text { comparativo } \\
(46,47)\end{array}$ & $\begin{array}{l}\text { Candidemia o } \\
\text { candidiasis } \\
\text { invasora }\end{array}$ & $\begin{array}{l}1 \\
1\end{array}$ & $\begin{array}{c}100 \text { (carga) } \\
\text { luego 50, } \\
150 \text { (carga) } \\
\text { luego } 75, \\
\text { y } 200 \\
\text { (carga) } \\
\text { luego } 100\end{array}$ & ) & $\begin{array}{l}\text { Respuesta global } \\
88 \%(200 / 100) \\
89 \%(150 / 75) \\
\text { y } 81 \%(100 / 50)\end{array}$ & $\begin{array}{l}\text { Discreta mejor respues- } \\
\text { ta a dosis más altas }\end{array}$ \\
\hline $\begin{array}{l}\text { Terapia de rescate } \\
\text { (41) }\end{array}$ & $\begin{array}{l}\text { Candidiasis } \\
\text { orofaríngea y } \\
\text { esofágica }\end{array}$ & 5 & $\begin{array}{c}100 \\
\text { (carga) y } \\
\text { luego } 50\end{array}$ & No & Éxito clínico 5/5 & Estudio muy pequeño \\
\hline
\end{tabular}

Además en el subgrupo de pacientes con neutropenia severa persistente la tasa de éxito fue pobre (menor del 20\%). Esta respuesta subóptima durante la neutropenia persistente es similar a la obtenida con anfotericina B, si bien existen ejemplos publicados en pacientes con neutropenia prolongada que responden a itraconazol o voriconazol $^{53,54}$.

Dos estudios (Tabla 6) con micafungina en aspergilosis se han realizado, el primero más amplio (290 pacientes) se realizó en aspergilosis invasora ${ }^{55,56}$ y otro en diversas formas de aspergilosis, llevado a cabo en Japón ${ }^{57}$. En la mayoría de los pacientes, micafungina fue agregada al tratamiento existente si el paciente no respondía. La dosis de micafungina varió de 50 a 300 mg diarios, y el investigador podía aumentar si la respuesta no se consideraba adecuada. Un revisor independiente de los casos establecía la certeza diagnóstica y los resultados, y la inclusión sólo de aquellos con aspergilosis invasora confirmada, que recibieron al menos siete días de tratamiento con micafungina, dejando 179 casos para el análisis de respuesta. La tasa de respuesta global fue de $37 \%$, con $40 \%$ para el grupo de terapia exclusiva con micafungina ${ }^{56}$. No existen datos actuales del uso de anidulafungina en aspergilosis.
Por lo anterior, el actual rol de las equinocandinas en el tratamiento de la aspergilosis invasora actualmente es difícil de definir. Se ha visto una buena eficacia y por cierto parecen representar una alternativa de terapia cuando existen efectos tóxicos frente a otros fármacos o éstos han fracasado. $\mathrm{Si}$ acaso las equinocandinas pudieran constituirse como terapia de primera línea versus voriconazol $^{59}$ es incierto, pues ciertos datos sugieren que serían menos efectivas en pacientes neutropénicos.

Fujisawa desarrolló un extenso estudio de profilaxis con miras al registro de micafungina ${ }^{60}$. En total 882 pacientes sometidos a transplante de células progenitoras hematopoyéticas fueron asignados en forma aleatoria a micafungina $50 \mathrm{mg} /$ día o fluconazol $400 \mathrm{mg} /$ día, durante más de 6 semanas. Se evaluó la presencia de infecciones que surgieron inclusive 30 días después de la profilaxis. El criterio de evaluación fue la sospecha de infección fúngica, y la terapia para esta infección fue anfotericina B. La tasa de respuesta a micafungina fue mejor que la de fluconazol (80 vs 73,5\%; $\mathrm{p}=0,025)$ y este beneficio se vio en todos los grupos de pacientes, incluyendo niños y ancianos, receptores de trasplante alogénico o autólogo, y pacientes con neutropenia persistente. De esta forma, y a pesar de la baja 
dosis de micafungina empleada, el beneficio quedó claramente demostrado. La tasa de aspergilosis documentada fue baja. Futuros estudios deberán demostrar si lo beneficios en reducción de mortalidad vistos con fluconazol ${ }^{61}$ se verán con micafungina.

La terapia combinada es una opción interesante para aquellos pacientes con pobre respuesta clínica a los actuales antifúngicos. Este punto ha sido ampliamente revisado para aspergilosis, el principal foco de interés en relación a equinocandinas $^{62}$. No se le ha descrito antagonismo in vivo ni in vitro. Tanto la combinación de caspofungina con anfotericina $\mathrm{B}$ en pacientes con cáncer $^{63}$ como la de caspofungina con itraconazol ${ }^{64}$ parecen ser promisorias. La tasa de respuesta en terapia combinada fue inferior que en aquellos con monoterapia de micafungina ${ }^{57}$. Este hallazgo indica probablemente la condición actual o anticipada del paciente y muestra la necesidad de estudios que evalúen si la terapia combinada es en el fondo desventajosa. La terapia combinada debiera ser apropiada para pacientes muy enfermos con predecible mal pronóstico.

\section{Eventos adversos y toxicidad}

Las equinocandinas han mostrado pocos efectos adversos y toxicidad. La máxima dosis tolerada de caspofungina en ratas es menor de 38 $\mathrm{mg} / \mathrm{kg}^{21}$ este dato no esta disponible para micafungina $o$ anidulafungina.

Los efectos adversos de grupo se ven en la Tabla 8, la reacciones de liberación de histamina son relativamente frecuentes dado su composición polipeptídica. Sin embargo, tales reacciones no se ven después de la administración de caspofungina o micafungina, pero pueden verse si la administración de anidulafungina es muy rápida.

La irritación local en el sitio de infusión es un problema común de los medicamentos bajo estudio clínico. La incidencia para caspofungina es

\section{Tabla 8. Efectos adversos de las equinocandinas}

$$
\begin{aligned}
& \text { Efecto de clase } \\
& \text { - Cefalea } \\
& \text { - Fiebre } \\
& \text { - Efectos tóxicos hepáticos } \\
& \text { - Flebitis } \\
& \text { - Reacciones de liberación de histamina } \\
& \text { - Hemólisis }
\end{aligned}
$$

\section{Otros efectos}

$$
\text { - Rash }
$$

cercana a $16 \%$, no habiéndose reportado para micafungina. Otro efecto común para caspofungina y micafungina es la alteración de las pruebas hepáticas, algo mayor para el primer fármaco sobre todo al combinarse con ciclosporina ${ }^{21,42}$. La fiebre es un efecto adverso frecuente del tratamiento con caspofungina, pudiendo alcanzar una incidencia de hasta $35 \%^{21,33,65}$. En cambio sólo $1 \%$ de los pacientes tratados con micafungina evidencia fiebre. La cefalea es un efecto adverso frecuente del grupo, 3\% durante el empleo de micafungina y $15 \%$ con caspofungina.

Así el perfil de seguridad de las tres equinocandinas es favorable, y mucho mejor que el de anfotericina B, ya sea liposomal (o lipídica) o no. Tal vez caspofungina tendría un menor margen terapéutico, en relación a las alteraciones hepáticas y el uso concomitante de ciclosporina, que los otros dos fármacos. Dada su farmacocinética predecible en las diferentes poblaciones de pacientes, son poco probable los efectos tóxicos dosis relacionados.

Finalmente, a la luz de los datos expuestos, podemos afirmar que las equinocandinas emergen como una alternativa concreta y segura frente a anfotericina B en aquellos pacientes con candidiasis o aspergilosis que cursen con una falla renal previa o desencadenada por el uso de anfotericina. Datos complementarios sobre equinocandinas pueden ser consultados en las revisiones de Maertens ${ }^{19}$, Chandrasekar $^{73}$ y Kartosins $^{74}$ relativas a caspofunginas, de Fromtling ${ }^{75}$ y Pawlitz $^{76}$, sobre micafungina y de Mazuelos ${ }^{58}$ sobre anidulafungina.

\section{Resumen}

La familia de lipopéptidos conocidos como equinocandinas emerge como las nuevas "penicilinas antifúngicas", capaces de destruir la pared celular micótica al inhibir la síntesis del glucano, fundamental constituyente de la estructura fúngica. Las equinocandinas han mostrado in vitro e in vivo, ser fungicidas contra la mayoría de las especies de Candida y fungistáticas contra Aspergillus sp, sin exhibir acción sobre células de mamíferos. Tres agentes emergen como los principales representantes de esta clase; caspofungina, micafungina y anidulafungina, estando las dos primeras ya licenciadas para su uso en humanos. Su óptimo perfil de seguridad, con baja incidencia y severidad de efectos adversos, cómoda posología y pocas interacciones con otros fármacos, representan notables ventajas para la terapéutica antifúngica moderna. Comparativamente han mostrado tener eficacia clínica similar a anfotericina $\mathrm{B}$, sin la toxicidad que este polieno tradicionalmente muestra, lo que sumado a la ausencia 
de antagonismo con otros antifúngicos permite sugerir que la terapia combinada pudiera ser un nuevo estándar de manejo para la tan temida aspergilosis invasora.

\section{Agradecimientos}

Agradezco a Estrella Martín Mazuelos por la información generosamente aportada sobre anidulafungina.

\section{Bibliografía}

1.- Groll A H, Shah P M, Mentzel C, Schneider M, JustNuebling G, Huebner K. Trends in the postmortem epidemiology of invasive fungal infections at a university hospital. J Infect 1996; 33: 23-32.

2.- Vogeser M, Haas A, Aust D, Ruckdeschel G. Postmortem analysis of invasive aspergillosis in a tertiary care hospital. Eur J Clin Microbiol Infect Dis 1997; 16: 1-6.

3.- Ribaud P, Chastang C, Latge J P, Baffroy-Laffitte L, Parquet N, Devergie A et al. Outcome and prognostic factors of invasive aspergillosis after allogeneic bone marrow transplantation. Clin Infect Dis 1999; 28: 32230 .

4.- Denning D W. Invasive aspergillosis. Clin Infect Dis 1998; 26: 781-805.

5.- Moore R D, Chaisson R E. Natural history of opportunistic disease in an HIV-infected urban clinical cohort. Ann Intern Med 1996; 124: 633-42.

6.- Lucero Y, Brucher R, Álvarez A M, Becker A, Cofré J, Enríquez $\mathrm{N}$ et al. Infección micótica profunda en niños con cáncer, neutropenia y fiebre, en Chile. Rev Méd Chile 2002; 130 (10): 1139-46.

7.- Wolff M, Diomedi A, Morales O, Bidart T, Dabanch J, Bustamante C, Northland R. Seguimiento prospectivo de una población infectada por VIH con y sin posibilidades de terapia anti-retroviral: impacto en sobrevida y complicaciones. Rev Méd Chile 2001; 129 (8): 886-94.

8.- Moore C B, Sayers N, Mosquero J, Slaven J, Denning D W. Antifungal drug resistance in Aspergillus. J Infect 2000; 41: 203-20.

9.- Sanglard D, Odds F C. Resistance of Candida species to antifungal agents: molecular mechanisms and clinical consequences. Lancet Infect Dis 2002; 2: 73-85.

10.- Nyfeler R, Keller S W. Metabolites of microorganisms, 143: echinocandin B, a novel polypeptide-antibiotic from Aspergillus nidulans var echinulatus--isolation and structural components. Helv Chim Acta 1974; 57: 2459-77.

11.- Masurekar P S, Fountoulakis J M, Hallada T C, Sosa M S, Kaplan L. Pneumocandins from Zalerion arboricola, II: modification of product spectrum by mutation and medium manipulation. J Antibiot (Tokyo) 1992; 45: 1867-74.

12.- Iwamoto T, Fujie A, Nitta K, Hashimoto S, Okuhara M, Kohsaka M. WF11899A, B and C, novel antifungal lipopeptides, I: taxonomy, fermentation, isolation and physico-chemical properties. J Antibiot (Tokyo) 1994; 47: 1084-91.

13.- Current W L, Tang J, Boylan C, Watson P, Zeckner $\mathrm{W}$, Turner $\mathrm{W}$ et al. Glucan biosynthesis as a target for antifungals: the echinocandin class of antifungal drugs. In: Dixon FK, Copping LG, Holloman DW, eds. The discovery and mode of action of antifungal drugs. Oxford: Bios Scientific, 1995: 143.

14.- Kurtz M B, Rex J H. Glucan synthase inhibitors as antifungal agents. Adv Protein Chem 2001; 56: 423-75.

15.- Cabello M A, Platas G, Collado J, Diez M T, Martín I, Vicente $\mathrm{F}$ et al. Arundifungin, a novel antifungal compound produced by fungi: biological activity and taxonomy of the producing organisms. Int Microbiol 2001; 4: 93-102.

16.- Ohyama T, Kurihara Y, Ono Y, Ishikawa T, Miyakoshi S, Hamano K et al. Aborcandins A, B, C, D, E and F, novel 1,3-beta-glucan synthase inhibitors: production and biological activity. J Antibiot 2000; 53: 1108-16.

17.- Pelaez F, Cabello A, Platas G, Diez M T, González del Val A, Basilio A et al. The discovery of enfumafungin, a novel antifungal compound produced by endophytic Hormonema species. Biological activity and taxonomy of the producing organisms. Syst Appl Microbiol 2000; 23: 333-43.

18.- Douglas C. Fungal beta (1,3)-D-glucan synthesis. Med Mycol 2001; 39: (suppl 1) 55-66.

19.- Maertens J, Boogaerts M. Caspofungina in the treatment of candidosis and aspergillosis. Int $\mathrm{J}$ Infect Dis 2003; 7: 94-101.

20.- Lucas R, De Sante K, Hatcher B, Heminway J, Lachno $\mathrm{R}$, Brooks $\mathrm{S}$ et al. LY303366 single dose pharmacokinetics and safety in healthy volunteers. Program and Abstracts of the 36rd Annual Interscience Conference on Antimicrobial Agents and Chemotherapy; September 15-18, 1996; New Orleans, American Society of Microbiology: abstr F-50.

21.- Caspofungin acetate. FDA advisory committee meeting background. http://www.fda.gov/ohrms/dockets/ ac/01/briefing/3676b1_02.pdf (accedido en Dec 1, 2003).

22.- Van der Horst C M, Saag M S, Cloud G A, Knopper J L, Bossuyt P M et al. Treatment of cryptococcal meningitis associated with the acquired immunodeficiency syndrome. N Engl J Med 1997; 337: 15-21.

23.- Holland S, Stone J, Li S, Wickersham P, Deutsch P, Winchell $G$ et al. Effect of hepatic insufficiency on the pharmacokinetics of caspofungin. Program and Abstracts of the 41th Annual Interscience Conference on Antimicrobial Agents and Chemotherapy; December 16-19, 2001; Chicago, American Society of Microbiology: abstr A-14.

24.- Walsh T J, Adamson P C, Seibel N L, Flynn P, Neely M, Miller A et al. Pharmacokinetics of caspofungin in paediatric patients. Program and Abstracts of the 42th Annual Interscience Conference on Antimicrobial Agents and Chemotherapy; September 27-30, 2002; San Diego, American Society of Microbiology: abstr M-896.

25.- Schwartz C, Seibel N, Arriet A, Flynn P, Shad A, Albano E et al. A phase 1 study to determine the safety and pharmacokinetics of FK463 (echinocandin) in febrile neutropenic pediatric patients. Program and Abstracts of the 40th Annual Interscience Conference on Antimicrobial Agents and Chemotherapy; September 16-20, 2000; Toronto, Canada, American Society of Microbiology: abstr 18.

26.- Thompson Micromedex Healthcare Series 2003; 119 (D).

27.- Holland S, Stone J, Wickersham P, Deutsch P, Winchell G, Herney $\mathrm{M}$ et al. Drug interactions between caspofungin and tacrolimus. Program and Abstracts of the 41th Annual Interscience Conference on Antimicrobial Agents and Chemotherapy; December 16-19, 2001; Chicago, American Society of Microbiology: abstr A-13. 
28.- Del Poeta M, Cruz M C, Cardenas M E, Perfect J R, Heitman J. Synergistic antifungal activities of bafilomycin A(1), fluconazole, and the pneumocandin MK-0991/caspofungin acetate (L-743,873) with calcineurin inhibitors FK506 and L-685,818 against Cryptococcus neoformans. Antimicrob Agents Chemother 2000; 44: 739-46.

29.- Townsend R, Hebert M, Wisemandle W, Bekersky H. Concomitant pharmacokinetics of micafungin, an echinocandin antifungal, and tacrolimus in healthy volunteers. Proceedings of the American College of Clinical Pharmacology 31st Annual Meeting; Sept 2123, 2002; San Francisco, USA: abstr 18.

30.- Thye D, Kilfoil T, Kilfoil G, Henkel T. Anidulafungin: safety and pharmacokinetics in subjects receiving concomitant cyclosporine. Program and Abstracts of the 42th Annual Interscience Conference on Antimicrobial Agents and Chemotherapy; September 27-30, 2002; San Diego, American Society of Microbiology: abstr A-1836.

31.- Denning W D. Echinocandin antifungal drugs. Lancet 2002; 362: 1142-51.

32.- Villanueva A, Arathoon E G, Gotuzzo E, Berman R S, DiNubile M J, Sable C A. A randomized double-blind study of caspofungin versus amphotericin for the treatment of candidal esophagitis. Clin Infect Dis 2001; 33: $1529-35$.

33.- Arathoon E G, Gotuzzo E, Noriega L M, Berman R S, DiNubile M J, Sable C A. Randomized, double-blind, multicenter study of caspofungin versus amphotericin $\mathrm{B}$ for treatment of oropharyngeal and esophageal candidiasis. Antimicrob Agents Chemother 2002; 46: 451-57.

34.- Pettengell K, Mynhardt J, Kluyts T, Simjee A, Baraldi E. A multicenter study of the echinocandin antifungal FK463 for the treatment of esophageal candidiasis in HIV positive patients. Program and Abstracts of the 40th Annual Interscience Conference on Antimicrobial Agents and Chemotherapy; September 16-20, 2000; Toronto, Canada, American Society of Microbiology: 371 (abstr 1104).

35.- Ally R, Schurmann D, Kreisel W, et al. A randomized, double-blind, double-dummy, multicenter trial of voriconazole and fluconazole in the treatment of esophageal candidiasis in immunocompromised patients. Clin Infect Dis 2001; 33: 1447-54.

36.- Brown G L, White R J, Turik M. Phase II, randomized, open label study of two intravenous dosing regimens of V-echinocandin in the treatment of esophageal candidiasis. Program and Abstracts of the 40th Annual Interscience Conference on Antimicrobial Agents and Chemotherapy; September 17-20, 2000, Toronto, Canada, American Society of Microbiology p 371: abstr 1106

37.- Krause D S, Henkel T, Goldstein B P, Walsh T J. Anidulafungin vs Fluconazole in esophageal Candidiasis: a phase 3 , randomized, double-blind, multicenter trial. 43rd Program and Abstracts of the 43th Annual Interscience Conference on Antimicrobial Agents and Chemotherapy, September 14-17, 2003, Chicago: 477: abstr $\mathrm{m} 1760$.

38.- Krause D, Birmingham W, Schranz J. Safety results from a phase 3, randomized, double-blind, doubledummy study of Anidulafungin vs Fluconazole in patients with esophageal candidiasis. 41st Meeting of Infectious Diseases Society of America Abstracts, October, 2003, page 49: abstr 136.

39.- Schranz J, Krause D, Henkel T. Clinical efficacy results from a phase 3 , randomized, double-blind, doubledummy study of anidulafungin $v s$ fluconazole in patients with esophageal candidiasis. $9^{\text {th }}$ Congress of European Confederation of Medical Mycology, October, 2003: 72: abstr O2.06.

40.- Kartsonis N, DiNubile M J, Bartizal K, Hicks P S, Ryan D, Sable C A. Efficacy of caspofungin in the treatment of esophageal candidiasis resistant to fluconazole. J Acquir Immun Defic Syndr 2002; 31: 183-7.

41.- Schranz J, Krause D, Goldstein B P, Henkel T. Efficacy results from a phase $2 / 3$, open-label study of anidulafungin in patients with fluconazole refractory mucosal candidiasis. $9^{\text {th }}$ Congress of European Confederation of Medical Mycology, October, 2003, Page 142: abstr P044.

42.- Mora-Duarte, Betts R, Rotstein C, Colombo A L, Thompson-Moya L, Smietana J et al. Comparison of caspofungin and amphotericin $\mathrm{B}$ for invasive candidiasis. N Engl J Med 2002; 347: 2020-9.

43.- Phillips P, Shafran S, Garber G, Rotstein Csmaill F, Fong I et al. Multicenter randomized trial of fluconazole versus amphotericin B for treatment of candidemia in non-neutropenic patients. Eur J Clin Microbiol Infect Dis 1997; 16: 337-45.

44.- Rex J H, Bennett J E, Sugar A M, Pappas P G, Van der Horst $\mathrm{C}$ M, Edwards $\mathrm{J}$ E et al. A randomized trial comparing fluconazole with amphotericin $\mathrm{B}$ for the treatment of candidemia in patients without neutropenia. N Engl J Med 1994; 331: 1325-30.

45.- Reush M, Fujisawa citado en 31.

46.- Krause D, Goldstein B, Wible M, Kilfoil G, Henkel T. A phase 2 dose-ranging study of the safety and efficacy of anidulafungin in invasive candidiasis (abstract). Proceedings of the 13th European Congress of Clinical Microbiology and Infectious Diseases; May 10-13, 2003; Glasgow, UK: 73 (abstr 403).

47.- Schranz J A, Krause D, Goldstein B P, Henkel T. Efficacy of Anidulafungin (ANID) for the treatment of candidemia. Program and Abstracts of the 43rd Annual Interscience Conference on Antimicrobial Agents and Chemotherapy, September 14-17, 2003, Chicago, page 448: abstr m971.

48.- Kartsonis N, Dinubile M, Bradshaw S, Lipka J, Sable C. Caspofungin for treatment of candida urinary tract infections: Review of the CAS Clinical Database. 41st Meeting of Infectious Diseases Society of America abstracts, October, 2003, page 49: abstr 135 .

49.- Sora F, Chiusolo P, Piccirillo N, Pagano L, Laurenti L, Farina $\mathrm{G}$ et al. Successful treatment with Caspofungin of hepatosplenic candidiasis resistant to liposomal amphotericin B. Clin Infect Dis 2002; 35: 1135-6.

50.- Lejko-Zupanc T, Mocina E, Vrevc F. Caspofungin as salvage treatment for Candida glabrata prosthetic hip infection. $9^{\text {th }}$ Congress of European Confederation of Medical Mycology, October, 2003, Page 179: abstr P082.

51.- Presterl E, Kotzalias N, Binder T, Mueller C, Willinger B, Graninger W. Successful treatment of Candida albicans endocarditis with Caspofungin. $9^{\text {th }}$ Congress of European Confederation of Medical Mycology, October, 2003, Page 186: abstr P090.

52.- Maertens J, Raad I, Petrikkos G, Selleslag D, Petersen $\mathrm{F}$, Sable $\mathrm{C}$ et al. Update of the multicenter noncomparative study of caspofungin (CAS) in adults with invasive aspergillosis (IA) refractory (R) or intolerant (I) to other antifungal agents: analysis of 90 patients. Program and Abstracts of the 42th Annual Interscience Conference on Antimicrobial Agents and Chemothe- 
rapy; September 27-30, 2002; San Diego, USA: 388 (abstr M-686).

53.- Denning D W, Lee J Y, Hostetler J S, Pappas P G, Kauffman C A, Dewsnup D H et al. NIAID mycoses study group multicenter trial of oral itraconazole therapy for invasive aspergillosis. Am J Med 1994; 97: 135-44.

54.- Denning D W, Ribaud P, Milpied N, Caillot D, Herbrecht R, Thiel E et al. Efficacy and safety of voriconazole in the treatment of acute invasive aspergillosis. Clin Infect Dis 2002; 34: 563-71.

55.- Ratanatharathorn V, Flynn P, van Burik J A, McSweeney P, Niederwieser D, Kontoyiannis D. Micafungin in combination with systemic antifungal agents in the treatment of refractory aspergillosis (RA) in bone marrow transplant (BMT) patients; Proceedings of the 44th Annual American Society of Hematology Meeting; Dec 6-10, 2002, Philadelphia, PA, USA: abstr 722 .

56.- Ullmann A J, Van Burik J A, McSweeney P, Ratanatharathorn V, Raymond J, de Morais V L et al. An open phase II study of the efficacy of micafungin (FK463) alone and in combination for the treatment of invasive aspergillosis (IA) in adults and children. Proceedings of the 13th European Congress of Clinical Microbiology and Infectious Diseases; 2003 May 1013; Glasgow, UK: abstr 722.

57.- Kohno S, Masaoka T, Yamaguchi H. A multicenter, open-label clinical study of FK463 in patients with deep mycosis in Japan. Program and Abstracts of the 41th Annual Interscience Conference on Antimicrobial Agents and Chemotherapy; 2001 December 16-19; Chicago, USA: p 384 (abstr J-834).

58.- Mazuelos M E. Anidulafungin Vincuron. Idrugs 2003; 6 (10): 980-6.

59.- Herbrecht R, Denning D W, Patterson T F, Bennett J E, Greene R E, Oestmann J M et al. Voriconazole versus amphotericin $\mathrm{B}$ for primary therapy of invasive aspergillosis. N Engl J Med 2002; 347: 408-15.

60.- Van Burik J, Ratanatharathorn V, Lipton J, Miller C, Bunin N, Walsh T J. Randomized, double-blind trial of micafungin versus fluconazole for prophylaxis of invasive fungal infections in patients undergoing haematopoietic stem cell transplant. Program and Abstracts of the 42th Annual Interscience Conference on Antimicrobial Agents and Chemotherapy; September 27-30, 2002, San Diego, USA: 401 (abstr M-1238).

61.- Marr K A, Seidel K, Slavin M A et al. Prolonged fluconazole prophylaxis is associated with persistent protection against candidiasis-related death in allogeneic marrow transplant recipients: long-term follow-up of a randomized, placebo-controlled trial. Blood 2000; 96: 2055-61.

62.- Steinbach W J, Stevens D A, Denning D W. Combination and sequential antifungal therapy for invasive aspergillosis: review of published in vitro and in vivo interactions and 6281 clinical cases from 1966 to 2002. Clin Infect Dis 2003; 37: (suppl 3) S188-224.

63.- Aliff T B, Maslak P G, Jurcic J G, Heaney M L, Cathcart K N, Sepkowitz K A et al. Refractory Aspergillus pneumonia in patients with acute leukemia. Cancer 2003; 97: 1025-32.

64.- Rubin M A, Carroll K C, Cahill B C. Caspofungin in combination with itraconazole for the treatment of invasive aspergillosis in humans. Clin Infect Dis 2002; 34: 1160-1.

65.- Villanueva A, Gotuzzo E, Arathoon E G, Noriega L M, Kartsonic N A, Lupinacci R J et al. A randomized double-blind study of caspofungin versus fluconazole for the treatment of esophageal candidiasis. Am J Med 2002; 113: 294-9.

66.- Sanz-Rodríguez C, Aguado J M, Cisneros J M, Vivancos R, González-Esteban J. Caspofungin therapy in documented fungal infections: Spanish experience before licensure of the drug. Program and Abstracts of the 40th Annual Interscience Conference on Antimicrobial Agents and Chemotherapy, American Society for Microbiology, September 27 - 30, 2002, San Diego, CA, page 394: abstr 895.

67.- Kartsonis N, Saah A, Lipka J, Taylor A, Sable C. Salvage therapy $(\mathrm{Rx})$ with Caspofungin for Invasive Aspergillosis: Results from the CAS Compassionate Use Study. Program and Abstracts of the 43rd ICAAC, American Society for Microbiology, September, 2003, page 451: abstr m-981.

68.- Walsh T, Sable C, Depauw B, Donowitz G, Maertens J, Baden $\mathrm{L}$ et al. A randomized, double-blind, multicenter trial of caspofungin $v s$ liposomal amphotericin B for empirical antifungal therapy of persistently febrile neutropenic patients. Program and Abstracts of the 43rd ICAAC, American Society for Microbiology, September 14-17, 2003, page 477: abstr m1761.

69.- Mattiuzzi G N, Kantarjian H, Alvarado G, Kontoyiannis D, Pierce S, Cortés $J$ et al. Intravenous Itraconazole $V s$ Caspofungin prophylaxis in patients with acute myelogenous leukemia and myelodysplastic syndrome undergoing induction chemotherapy. Program and Abstracts of the 43rd Annual Interscience Conference on Antimicrobial Agents and Chemotherapy, American Society for Microbiology, September 14-17, 2003, page 451: abstr m984.

70.- Suleiman J, Della Negra M, Llanos-Cuentas A, Ticona E, Rex J H, Buell D N. Open label study of Micafungin in the treatment of esophageal candidiasis (EC). Program and Abstracts of the 42th Annual Interscience Conference on Antimicrobial Agents and Chemotherapy, American Society for Microbiology, September 27 - 30, 2002, San Diego, CA, page 394: abstr m892.

71.- De Wet N T E, Llanos-Cuentas A, Suleiman H, Baraldi E, Krantz E, Della Negra M et al. Micafungin (FK463) dose response and comparison with fluconazole in esophageal candidiasis. Program and Abstracts of the 43rd Annual Interscience Conference on Antimicrobial Agents and Chemotherapy, American Society for Microbiology, September 14-17, 2003, page 476: abstr m1754.

72.- Llanos Cuentas A, De Wet N, Suleiman J, Baraldi E, Krantz E F W, Della Negra M et al. Micafungin (FK463) dose response and comparison with fluconazole in oesophageal candidiasis. $9^{\text {th }}$ Congress of European Confederation of Medical Mycology, October, 2003, Page 68: abstr 02.02.

73.- Chandrasekar P H, Manavathu E K. Caspofungin. Drugs of Today 2002, 38 (12): 829-46.

74.- Kartsonis N A, Nielsen J, Douglas C M. Caspofungin: the first in a new class of antifungal agents. Drug Resistance Updates 6 2003; 197-218.

75.- Fromtling R. Micafungin sodium (FK-463). Drugs of Today 2002; 38 (4): 245-57.

76.- Pawlitz D, Young M, Klepser M. Micafungin: A new echinocandin antifungal. Formulary 2003; 38: 35467.

Correspondencia a:

Alexis Diomedi Pacheco.

E-mail: adiomedip@entelchile.net 